\title{
Plasmonics-high-speed photonics for co-integration with electronics
}

\section{Review Article}

\section{Author(s):}

Koch, Ueli (D); Uhl, Christopher; Hettrich, Horst; Fedoryshyn, Yuriy; Moor, David (ID; Baumann, Michael; Hoessbacher, Claudia; Heni, Wolfgang (D); Baeuerle, Benedikt; Bitachon, Bertold lan (D); Josten, Arne (ID; Ayata, Masafumi; Xu, Huajun; Elder, Delwin L.; Dalton, Larry R.; Mentovich, Elad; Bakopoulos, Paraskevas; Lischke, Stefan; Krüger, Andreas; Zimmermann, Lars; Tsiokos, Dimitris; Pleros, Nikos; Möller, Michael; Leuthold, Juerg (iD)

\section{Publication date:}

2021-05

\section{Permanent link:}

https://doi.org/10.3929/ethz-b-000485515

\section{Rights / license:}

Creative Commons Attribution 4.0 International

\section{Originally published in:}

Japanese Journal of Applied Physics 60, https://doi.org/10.35848/1347-4065/abef13

\section{Funding acknowledgement:}

688166 - A generic CMOS-compatible platform for co-integrated plasmonics/photonics/electronics PICs towards volume manufacturing of low energy, small size and high performance photonic devices (SBFI) 
Japanese Journal of Applied

Physics

PROGRESS REVIEW • OPEN ACCESS

Plasmonics-high-speed photonics for co-integration with electronics

To cite this article: Ueli Koch et al 2021 Jpn. J. Appl. Phys. 60 SB0806

View the article online for updates and enhancements. 


\title{
Plasmonics-high-speed photonics for co-integration with electronics
}

\author{
Ueli Koch ${ }^{1 *} \mathbb{0}$, Christopher Uhl' ${ }^{2}$, Horst Hettrich ${ }^{3}$, Yuriy Fedoryshyn ${ }^{1}$, David Moor ${ }^{1}$, Michael Baumann ${ }^{1}$, \\ Claudia Hoessbacher ${ }^{1,4}$, Wolfgang Heni ${ }^{1,4}$, Benedikt Baeuerle ${ }^{1,4}$, Bertold I. Bitachon ${ }^{1}$, Arne Josten ${ }^{1}$, Masafumi Ayata ${ }^{1}$, \\ Huajun Xu ${ }^{5}$, Delwin L. Elder ${ }^{5}$, Larry R. Dalton ${ }^{5}$, Elad Mentovich ${ }^{6}$, Paraskevas Bakopoulos ${ }^{6}$, Stefan Lischke ${ }^{7}$, \\ Andreas Krüger ${ }^{7}$, Lars Zimmermann ${ }^{7,8}$, Dimitris Tsiokos ${ }^{9}$, Nikos Pleros ${ }^{9}$, Michael Möller ${ }^{2,3}$, and Juerg Leuthold ${ }^{1,4}$ \\ ${ }^{1}$ ETH Zurich, Institute of Electromagnetic Fields (IEF), Zürich, Switzerland \\ ${ }^{2}$ Saarland University, Chair of Electronics and Circuits, Saarbrücken, Germany \\ ${ }^{3}$ MICRAM Microelectronic GmbH, Bochum, Germany \\ ${ }^{4}$ Polariton Technologies Ltd., Rüschlikon, Switzerland \\ ${ }^{5}$ University of Washington, Department of Chemistry, Seattle, WA, United States of America \\ ${ }^{6}$ NVIDIA networking, Yokneam, Israel \\ ${ }^{7}$ IHP_Leibniz-Institut für innovative Mikroelektronik, Frankfurt (Oder), Germany \\ ${ }^{8}$ Technische Universität Berlin, Institute of High-Frequency and Semiconductor System Technologies, Berlin, Germany \\ ${ }^{9}$ Aristotle University of Thessaloniki, Center for Interdisciplinary Research and Innovation, Thessaloniki, Greece \\ *E-mail: uelikoch@ethz.ch
}

Received January 15, 2021; revised February 22, 2021; accepted March 16, 2021; published online April 26, 2021

New high-speed photonic technologies and co-integration with electronics are required to keep up with the demand of future optical communication systems. In this paper, plasmonics is presented as one of the most promising next-generation photonic technologies that already fulfils these requirements in proof-of-concept demonstrations. Plasmonics features not only modulators and detectors of highest speed, but also compactness, cost- and energy-efficiency, and compatibility with CMOS electronics. Recently, co-integration with electronics was demonstrated with record performances of $222 \mathrm{GBd}$ in a hybrid InP electronic-plasmonic transmitter assembly and of $120 \mathrm{GBd}$ with a monolithic BiCMOS electronicplasmonic transmitter. (C) 2021 The Japan Society of Applied Physics

\section{Introduction}

Next-generation optical interconnects have to overcome the capacity gap between electronics and photonics. Current solutions are sufficient for links of tens or hundreds of $\mathrm{Gb} / \mathrm{s}$, but $\mathrm{Tb} / \mathrm{s}$ will soon be required in datacentres. ${ }^{1,2)}$ Hence, parallelisation and high data rates per line are of particular need. Only a compact, fast, cost- and energy-efficient platform can achieve this goal. ${ }^{3)}$ For this purpose, high-speed photonics has to catch up and electronic-photonic co-integration has to overcome the interface bottlenecks.

Currently, a variety of high-speed photonic technologies for transmitters and receivers based on indium phosphide (InP) photonics, ${ }^{4-7)}$ lithium niobate (LNB) photonics, ${ }^{8,9)}$ silicon (Si) photonics ${ }^{10-13)}$ or plasmonics $^{14-24)}$ are being investigated, while no leading technology could establish itself yet. Possible reasons for this are limited device performance, technology costs, manufacturing maturity or challenges in co-integration with electronics. For example, $\mathrm{Si}$ photonics $(\mathrm{SiPh})$ is already a mature technology with expected yearly revenues of 4 billion USD in $2025 .^{25)}$ Yet, its active components have large footprints, which renders scaling to higher speed a challenge. In contrast, plasmonics can easily reach highest speeds as the photonic technology of largest bandwidth ${ }^{21)}$ and ultra-low energy consumption. ${ }^{20)}$ Maturity and low costs in production can be achieved by combining plasmonics with well-developed photonics for passive components. Yet, all photonic technologies are still facing the co-integration challenge.

State-of-the-art electronic-photonic co-integration is based either on the hybrid assembly of separate chips in bond-wire or flip-chip configurations or on the monolithic integration on a common substrate. As a first option, bond-wire assemblies currently offer the highest-speed transmitters reaching symbol rates of $222 \mathrm{GBd}$ with plasmonics, ${ }^{23)} 192 \mathrm{GBd}$ with InP photonics ${ }^{26)}$ and $112 \mathrm{GBd}$ with Si photonics. ${ }^{13)}$ Yet, bandwidth limitations will eventually constrain further scaling. A second option is flip-chip assemblies, which offer reduced parasitics ${ }^{27)}$ and operation speeds of $100 \mathrm{GBd}$ using InP photonics. ${ }^{28)}$ Still, hybrid assemblies remain costly and hard to scale due to the technology interface at the highest symbol rate. Monolithic integration is expected to deliver a cost-efficient and high-performant alternative. Here, electronics and photonics are integrated as layers on the same substrate and connected through on-chip vias. Silicon-based zero-change CMOS photonics offers a complete data link at $10 \mathrm{GBd}^{29)}$ and transmitter operation at $40 \mathrm{GBd}^{30)}$ However, standard CMOS electronics are confronted with bandwidth limitations and alternative high-speed electronics such as InP or BiCMOS electronics are of particular interest. The latter achieves symbol rates of $180 \mathrm{GBd}^{31)}$ and was integrated with plasmonics in the currently fastest monolithic transmitter with data modulation at up to $120 \mathrm{GBd} .^{24)}$

In this progress review, the latest progress on co-integration of plasmonics with electronics is discussed. First, a brief overview over the plasmonic technology for optical communication is given. It is highlighted why plasmonics is particularly suited for high-speed co-integration. Second, the most common co-integration approaches are presented and two are selected for discussion in more detail at the example of electronic-plasmonic transmitters. A hybrid InP electronic-plasmonic transmitter using a bond-wire assembly reaches highest symbol rates of $222 \mathrm{GBd}^{23)}$ A monolithic BiCMOS electronic-plasmonic transmitter operating at 
$120 \mathrm{GBd}$ is the fastest and most compact of its kind. ${ }^{24)}$ Both show the technology's readiness for future optical communication systems.

The paper is in part based on the invited contribution at the 2020 International Conference on Solid State Devices and Materials (SSDM2020). ${ }^{32)}$

\section{Plasmonics-high-speed photonics}

Plasmonics as a high-speed photonic platform has recently and repeatedly raised attention due to its unique advantages such as speed and efficiency. Hence, active plasmonic structures such as modulators and detectors might become key components in future high-speed optical communication systems ranging from chip-to-chip over short-reach to longreach links. $^{3)}$

A brief conceptual overview over plasmonics shall be given before diving into its application for high-speed communications. Plasmonics is a subcategory of optics, which deals with electromagnetic waves coupled to charge oscillations on metal-dielectric interfaces, so-called surface plasmon polaritons (SPPs). The interaction of the electric and magnetic fields with the charges and vice versa is schematically shown in Fig. 1(a). The strong coupling binds the waves to the surface and leads to an exponential field decay perpendicular to it as depicted in Fig. 1(b). On one hand, when SPPs propagate along an open surface, the electromagnetic energy is transported along it and the structure acts as a plasmonic waveguide. On the other hand, when SPPs propagate along a closed surface, it can positively interfere with itself at a resonance frequency. A plasmonic standing wave is observed, which is called localised surface plasmon. Such a structure leads to strong localised fields and acts as plasmonic nano-antenna.

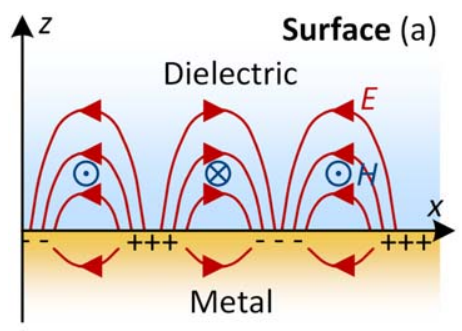

(c) Slab

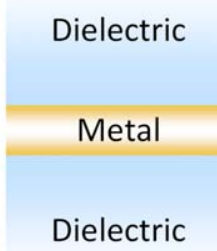

(d) Hybrid

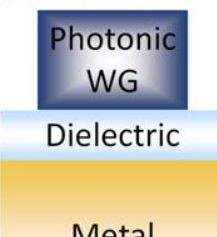

Metal

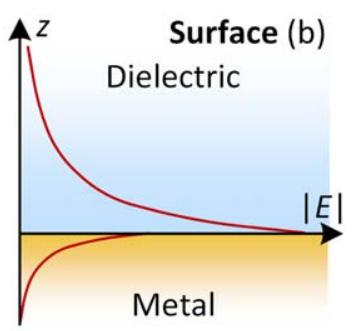

(e) Slot

$$
\text { Metal }
$$

Dielectric

Metal
Fig. 1. (Color online) Plasmonic waveguides. (a), (b) Surface plasmon polariton (SPP) propagation along a metallic surface. ${ }^{33)}$ (a) Interaction of the electric (red) and magnetic (blue) fields with the surface charges in the metal. Light is coupled to charge oscillations and propagates as electromagnetic wave along the surface. (b) Characteristic plasmonic exponential field decay away from the surface. The field is confined to the surface with typical penetration depths on the nanometre scale. (c) Plasmonic slab waveguide. The fields on both sides are coupled and guided along the metal surface. (d) Hybrid plasmonic waveguide. A waveguide-like photonic ridge on top of a plasmonic metal leads to hybrid photonic-plasmonic propagation. (e) Plasmonic slot waveguide. The guided wave is confined between the two metal films.
Over the last few decades, plasmonics raised a lot of attention thanks to the strong field enhancement by confining in space beyond the diffraction limit and reaching the nanometre scale. ${ }^{34-36)}$ Effects like extraordinary optical transmission, ${ }^{37,38)}$ surface-enhanced Raman scattering ${ }^{39,40)}$ and the creation of plasmonic waveguides ${ }^{41-47)}$ and nano-antennas ${ }^{48-50)}$ enabled a wide range of technologies for (bio-)sensing, ${ }^{51-53)}$ medicine ${ }^{54-57)}$ and integrated photonics. ${ }^{46,58,59)}$

In integrated photonics, a variety of plasmonic waveguides $^{41-47)}$ are available such as surface, slab, hybrid and slot waveguides, see Fig. 1, Surface waveguides ${ }^{44)}$ comprise optically thick metal films covered by a dielectric. The waves follow the SPP concept shown in Figs. 1(a)-1(b). Slab waveguides ${ }^{41)}$ are similar, but consist of a thin metal film sandwiched between two dielectrics as shown in Fig. 1(c). They are often referred to as insulator-metalinsulator waveguides and have coupled fields propagating on both sides of the metal. Surface and slab waveguides are usually rather weakly guiding, which gives less field enhancement but higher penetration into the dielectric and lower propagation loss. This makes them efficient for sensing and imaging applications, when scanning over a large volume. Slot and hybrid waveguides allow for stronger confinement beyond the dimensions of photonic counterparts. Hybrid plasmonic waveguides ${ }^{45)}$ are composed of a photonic waveguide-like part on top of a dielectric-coated metal film (or upside-down), see Fig. 1(d), and are also called dielectricloaded plasmonic waveguides. They are called hybrid because the guided mode is of photonic nature in the photonic part and of plasmonic nature in the metal part. The hybrid mode exhibits good field confinement in the dielectric layer, while still having low-loss propagation. Plasmonic slot waveguides $^{42,43)}$ as depicted in Fig. 1(e) offer the strongest confinement as light can be squeezed into an almost arbitrary small gap between two metals. ${ }^{35,36)}$ They are also called metal-insulator-metal waveguides and achieve the highest field enhancement among the presented structures. This can be efficiently exploited in applications such as lasers ${ }^{60,61)}$ and components for high-speed optical communications as reviewed below, yet comes at the price of increased propagation loss.

The choice of the metal for the plasmonic waveguide defines the basic properties of plasmonic devices such as the propagation loss. ${ }^{62,63)}$ Gold $(\mathrm{Au})$, silver $(\mathrm{Ag})$, copper $(\mathrm{Cu})$ and aluminium $(\mathrm{Al})$ are the standard plasmonic materials in the near-infrared. They have a permittivity with large negative real part, pushing the field out of the metal and into the active material (enhancing light-matter interaction), and a small imaginary part, reducing the absorption in the metal. Among them, silver has the lowest loss, but it suffers from quick degradation and resulting in worsened device performance. Therefore, gold is favourable because it still offers low loss and additionally inertness, which allows for efficient devices and compatibility with most fabrication processes. Yet, it is not available in CMOS processes such as copper and aluminium. ${ }^{64)}$ Hence, low-loss copper might be desirable once diffusion and oxidation are under control. In contrast, aluminium is better controllable in fabrication and operation. But, it has the highest losses among these materials, which must be compensated by more efficient light-matter interaction. 
When integrating plasmonics with conventional photonics, an additional converter structure is required. This can be efficiently implemented either by using a tapering structure ${ }^{65}$ or a directional coupling. ${ }^{66)}$ Measured excess losses were about $0.5 \mathrm{~dB}$ for a taper-like converter into a plasmonic slot waveguide $^{20)}$ and $0.3 \mathrm{~dB}$ for a directional coupler into a hybrid plasmonic waveguide. ${ }^{67)}$ Yet, integration with conventional photonics might not be necessary, when directly coupling from an optical fiber into a plasmonic device. ${ }^{15,19,68,69)}$ This renders additional photonic-plasmonic converters obsolete and makes plasmonic devices even more compact and integrable on a variety of substrates.

Plasmonics as a high-speed photonic platform ${ }^{14-24)}$ offers particular advantages over competing technologies, while few current limitations remain to be overcome. Typically, high-speed plasmonics resorts to slot waveguides for a variety of reasons. First, slot waveguides allow for strong confinement and high field intensity, which results in a strong light-matter interaction. Second, the plasmonic waveguide simultaneously serves as high-speed electrodes, which results in an optimal overlap between the electrical and optical fields. This renders nonlinear effects, as for example the electrooptical conversion in modulators, particularly (energy-)efficient. Third, plasmonic components are ultra-compact thanks to the beforementioned arguments. This significantly reduces both the contact resistance and the capacitance in such devices. In turn, the RC time constant drops and the electrical bandwidth increases to the highest values of their kind. ${ }^{21)}$ These advantages come at the price of plasmonic propagation loss. This fundamental loss of plasmonic devices is only about $5 \mathrm{~dB}$ in current demonstration and therefore already comparable to alternative high-speed active optical components. On top, it is expected that it can be further reduced by using advanced driving schemes ${ }^{22)}$ and by further optimising the nonlinear efficiency of the electro-optical material in the plasmonic slot. ${ }^{70,71)}$ Additional losses come from couplers and converters and are expected to reach similar values as in other technologies with the future integration of plasmonics in photonic fab processes.

Plasmonic high-speed modulators are versatile electrooptic components for the transmitter. They offer solutions for phase, intensity and amplitude modulation in simple phase ${ }^{72)}$ or advanced Mach-Zehnder (MZM) ${ }^{14,23)}$ ring, ${ }^{73)}$ or $\mathrm{IQ}^{20)}$ configurations. These modulators offer record symbol rates up to $222 \mathrm{GBd}^{23)}$ and data rates up to $400 \mathrm{~Gb} \mathrm{~s}^{-1}$ per optical carrier. ${ }^{20)}$ Key to the high speed is an electro-optical bandwidth exceeding $500 \mathrm{GHz}^{21)}$ This is inherent to the plasmonic modulator structure and the field confinement to the nanometre scale, see Fig. 2. The basic element is a plasmonic phase shifter (gold) as shown in the colourised scanning electron microscope image in Fig. 2(a). The modulation takes place in an organic ${ }^{70)}$ or ferroelectric ${ }^{17)}$ electro-optic material inside the narrow metal slot. Since the slot metals serve both as driver electrodes, see Fig. 2(b), and as plasmonic waveguide, see Fig. 2(c), both electrical fields are confined to the same area, which enables a strong lightmatter interaction. This reduces the requirements for size and energy, and renders plasmonic modulators fast, ${ }^{21)}$ compact $^{15,19,24,74)}$ and energy-efficient. ${ }^{20)}$ Latter allows for operation at CMOS-compatible voltages. ${ }^{22)}$ Furthermore, plasmonic modulators are not bound to photonic
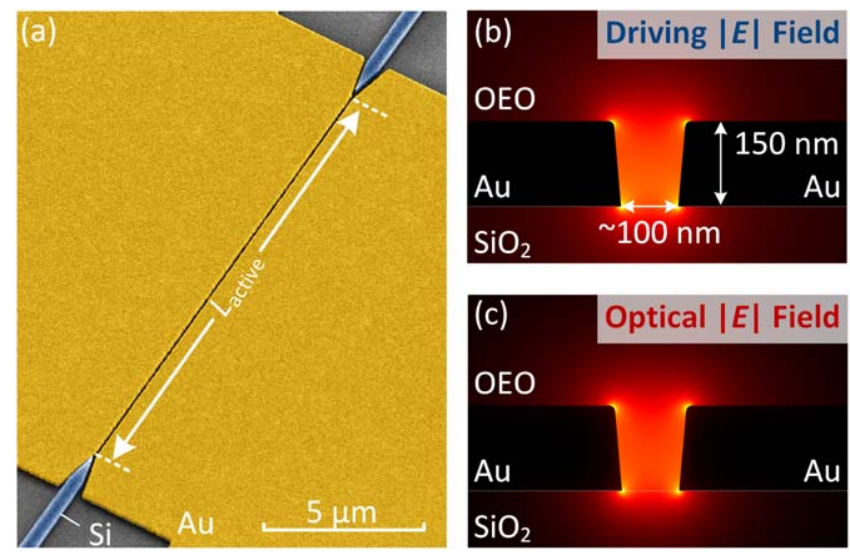

Fig. 2. (Color online) High-speed plasmonic modulator. ${ }^{20)}$ (a) Colourised SEM image of the modulator with the input and output silicon photonic waveguides (blue) and the active plasmonic slot (gold). (b), (c) Electrical field distributions. Both the (b) driving and (c) optical electrical field are well confined to the plasmonic slot, which renders the plasmonic modulator ultracompact, highly efficient and extremely broadband.

substrates ${ }^{15)}$ and allow for dense parallelisation in terabit arrays. ${ }^{19)}$ Hence, they promise great potential not only for hybrid $^{23)}$ but also for monolithic ${ }^{24)}$ integration with electronics.

Plasmonic high-speed detectors are the complementing opto-electric component for the receiver. Also in the detector, plasmonics allows for field confinement to the nanometre scale and hereby enhances the light-matter interaction. This leads to energy-efficient devices with high responsivity and compact footprints. ${ }^{75,76)}$ High-speed integrated plasmonic detectors based on germanium ${ }^{16)}$ or graphene ${ }^{18)}$ as absorbing medium show promising results. In proof-of-concept experiments with a single-layer graphene detector, ${ }^{18)}$ an optoelectric bandwidth beyond $110 \mathrm{GHz}$, an optical bandwidth above $140 \mathrm{~nm}$ in the C-band and a responsivity of $0.5 \mathrm{~A} / \mathrm{W}$ were measured. A data detection experiment demonstrated operation at a symbol rate of $100 \mathrm{GBd}$. Since plasmonic detectors are only a few micrometres in length, they are extremely compact and not bound to a photonic substrate, which makes them particularly suitable for co-integration with electronics.

\section{Co-integration with electronics}

Co-integration of electronics and photonics is one of the major challenges to advance in high-speed optical communication systems. Key to the success is optimising the interface between the electronic and photonic domains. While a variety of high-speed electronic and photonic solutions are available, a high-speed interface is remaining challenging.

The different approaches for co-integration from industrial state-of-the-art to research are shown in Fig. 3. Traditionally, electronics and photonics were treated separately. Individual modules were manufactured and assembled, see Fig. 3(a). Yet, connectorisation and cabling are not only limiting the scaling in speed but also became a significant cost factor. Copackaged optics are now replacing the traditional approach, where electronics and photonics are still on separate chips but assembled in a hybrid module. Two approaches of hybrid integration are hereby pursued. First, Fig. 3(b) shows a bondwire assembly where electronics and photonics are integrated 


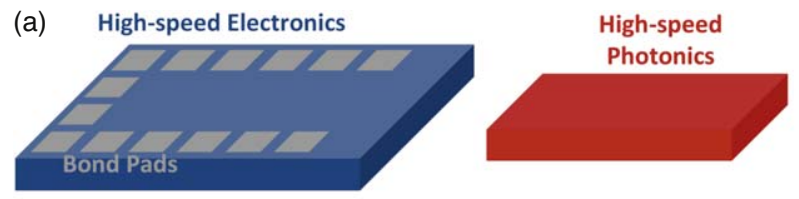

(b)

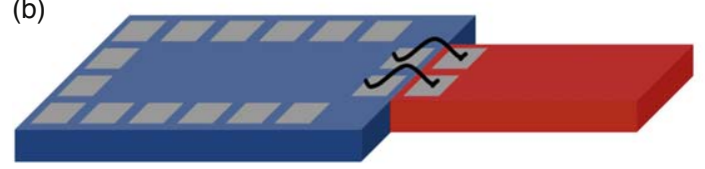

(c)

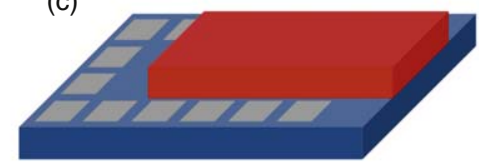

(10)

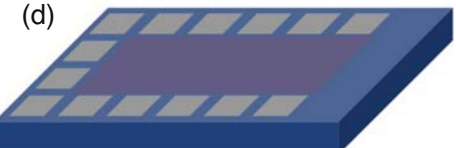

Fig. 3. (Color online) Co-integration approaches for high-speed electronics and photonics. (a) Completely separate chips. External equipment such as probes or cables are used for connection. (b) Bond-wire assembly. The two chips are assembled next to each other and connected at the high-speed interface with bond-wires. (c) Flip-chip assembly. The photonic chip is assembled on top of the electronic chips or vice versa. They are directly connected via the bond-pads on the individual chips. (d) Monolithic assembly. Both electronics and photonics are realised on a single substrate. No extra interface is required between the two domains.

side-by-side and connected by bond-wires. Second, Fig. 3(c) depicts a flip-chip assembly, where both technologies are integrated on top of each other and directly connected with bond-pads. In research, monolithic assemblies as shown in Fig. 3(d) are entering the focus of the community. Electronics and photonics are merged into a single technology, which promises highest speed and efficiency at lowest costs and on the smallest footprint. However, merging of electronics and photonics gives rise to all new challenges that have yet to be tackled.

\subsection{Hybrid integration}

Hybrid integration in a bond-wire assembly is the state-ofthe-art co-integration technology. Currently, it is offering the fastest transmitters thanks to its flexibility and the almost independent design and performance optimisation of the individual high-speed electronic and photonic components. Yet, the bandwidth-limited bond-wire interface will eventually prohibit further scaling due to signal degeneration.

A hybrid InP electronic-plasmonic transmitter demonstrated so far the highest transmitter speed with symbol rates up to $222 \mathrm{GBd}^{23)}$ The authors combined fastest electronics and photonics by assembling an InP electronic chip and a plasmonic chip side-by-side, connected by bond-wires, in a common module as shown in Fig. 4.

The InP electronics consists of a 2:1 digital selector (SEL) that generates an electrical output data signal by multiplexing two externally supplied input data streams. ${ }^{77)}$ The SEL achieves the high-speed performance thanks to the InP double-heterojunction bipolar transistor technology that achieves a transit frequency $f_{\mathrm{T}}=400 \mathrm{GHz}$. In stand-alone measurements, symbol rates up to 222 GBd were demonstrated.

The plasmonics comprises a silicon-based plasmonic modulator in a Mach-Zehnder configuration that generates an optical intensity modulation from the electrical driving signal. It uses $\mathrm{SiPh}$ grating couplers, waveguides and multimode interferometers to route the light on-chip and bring it to the phase modulators in the MZM arms. The modulators are realised in gold plasmonics with a nonlinear organic electrooptic material inside the slot and are operated in push-pull mode. They are connected as short as possible with the bondpads, which supply them with the electrical input signal.

Data modulation experiments of the complete bond-wire assembly at $222 \mathrm{GBd}$ demonstrated the performance of the

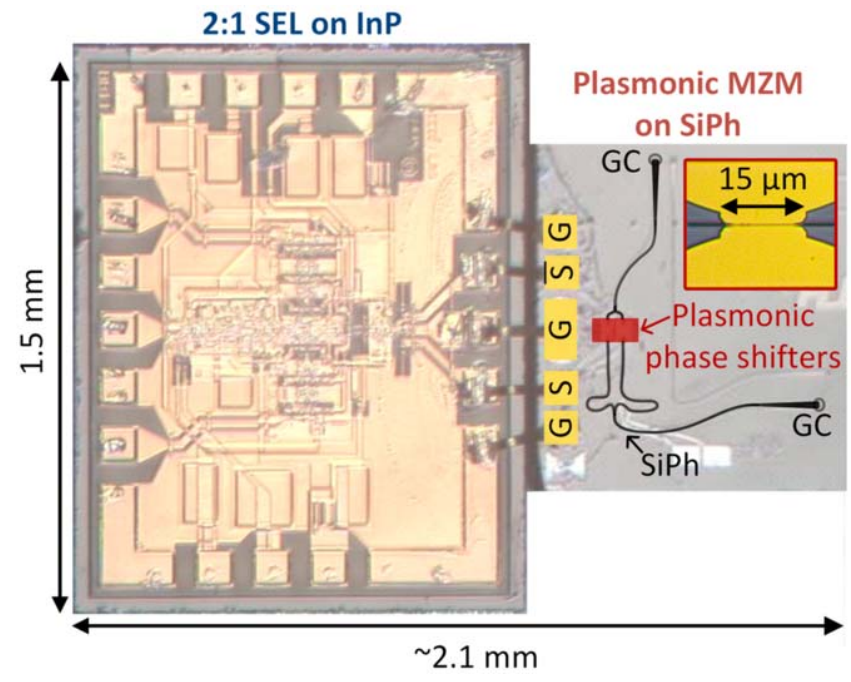

Fig. 4. (Color online) Hybrid electronic-plasmonic high-speed transmitter. ${ }^{23)}$ InP electronics perform a 2:1 multiplexing of high-speed electrical signals, which is fed to the output pads. Bond-wires connect the electronic chip with the silicon-plasmonic chip, where the electrical signal drives a silicon-based plasmonic Mach-Zehnder interferometer for intensity modulation.

hybrid transmitter. The received eye diagrams of a pure electrical and an electro-optical data modulation experiment are displayed in Fig. 5. To date, this marks the fastest transmitter independent of the co-integration scheme.

\subsection{Monolithic integration}

Monolithic integration is considered the most future-proof technology combining high-speed electronics and highbandwidth photonics on the same substrate. Hence, an interface of highest speed and lowest parasitics can be exploited. Furthermore, it is expected that monolithic

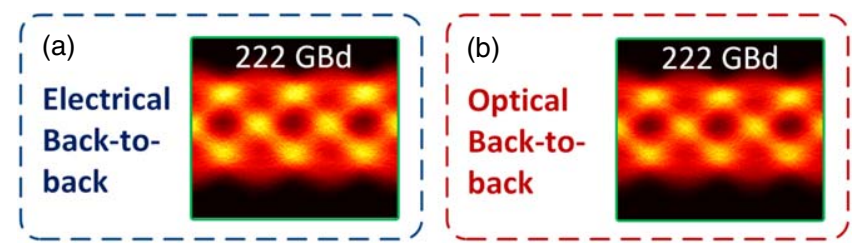

Fig. 5. (Color online) Received eye diagrams of the hybrid electronicplasmonic transmitter at $222 \mathrm{GBd}^{23)}$ (a) Electrical and (b) optical back-toback eye diagrams were measured. 


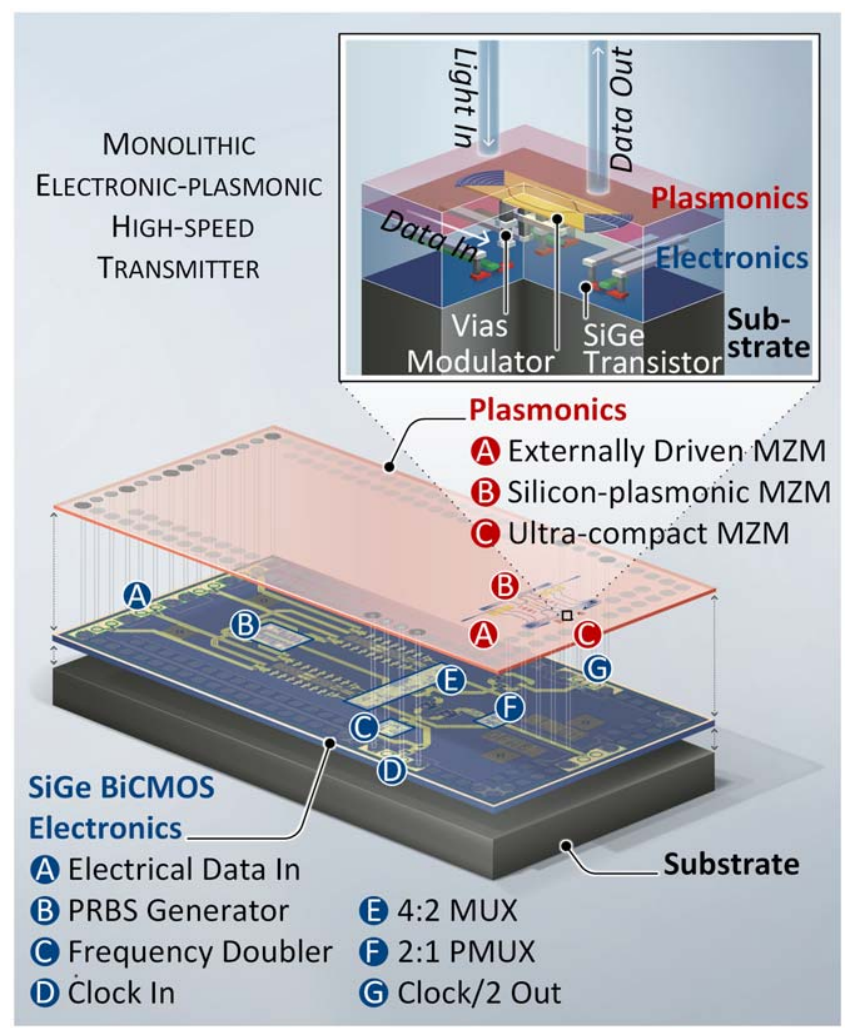

Fig. 6. (Color online) Monolithic electronic-plasmonic high-speed transmitter. ${ }^{24)} \mathrm{SiGe}$ BiCMOS electronics performs a 4:1 power multiplexing to generate a high-speed output signal to drive the plasmonic modulator. The plasmonic modulator converts the electrical signal into an optical intensity modulation for transmission over an optical fibre link. In particular, an ultracompact Mach-Zehnder modulator design with $29 \times 6 \mu \mathrm{m}^{2}$ footprint was introduced (see inset).

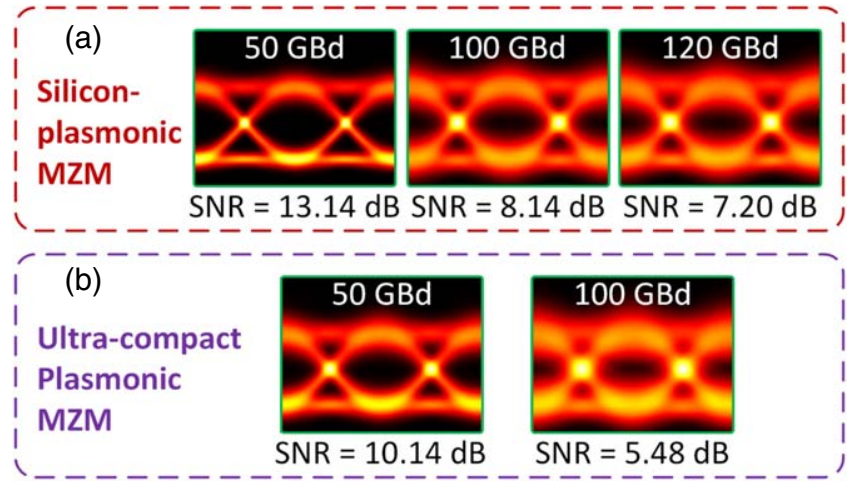

Fig. 7. (Color online) Received electro-optical eye diagrams of the monolithic BiCMOS electronic-plasmonic transmitter. ${ }^{24)} \mathrm{Up}$ to $120 \mathrm{GBd}$ with the silicon-plasmonic MZM and $100 \mathrm{GBd}$ with the ultra-compact plasmonic MZM were demonstrated, respectively.

integration significantly reduces the overall expenses from manufacturing to assembly and testing.

A monolithic bipolar CMOS electronic-plasmonic highspeed transmitter was recently demonstrated achieving symbol rates beyond $100 \mathrm{GBd}^{24)}$ To this end, one of the world's fastest electronic technologies and the photonics of highest bandwidth were monolithically integrated on a common substrate as depicted in Fig. 6.

In the SiGe BiCMOS electronic layers (blue), a high-speed data signal is generated through multiplexing that reaches total symbol rates of up to $180 \mathrm{GBd}^{31)}$ Four intermediate- speed electrical input signals are either externally provided or generated on chip. These input signals are then multiplexed in two stages, a 4:2 and a 2:1 stage, into the high-speed output signal. The 2:1 stage hereby replaces the standard driver amplifier and directly drives the output load. This is commonly called power multiplexing and provides the best signal quality and highest energy-efficiency at high speeds. ${ }^{78)}$ Note that BiCMOS electronics is particularly advantageous as it combines high-speed electronics with standard CMOS for control and data processing, while simultaneously featuring compatibility with Si photonics.

The plasmonic top layer (red) comprises all photonic devices. They consist of passive $\mathrm{Si}$ photonic components for coupling and guiding of light and active plasmonic components for electro-optic modulation. A Mach-Zehnder modulator (MZM) is chosen for intensity modulation at the transmitter and direct detection at the receiver. Two device concepts were investigated. First, a silicon-plasmonic MZM uses standard silicon passives for photonic on-chip routing and plasmonic phase shifters in the MZM arms. Second, an ultra-compact plasmonic MZM with a record-small footprint of $29 \times 6 \mu \mathrm{m}^{2}$ uses direct fibre-to-plasmonic couplers into an all-plasmonic MZM as shown in the inset of Fig. 6.

The monolithic transmitter performance was demonstrated in data modulation experiments with symbol rates up to $120 \mathrm{GBd}$ and operating under ambient air conditions. The measured electro-optical eye diagram for both modulator types are shown in Fig. 7. The silicon-plasmonic MZM reached up to $120 \mathrm{GBd}$ symbol rate and remains the fastest monolithic transmitter to-date. The ultra-compact plasmonic MZM reached up to $100 \mathrm{GBd}$ and persuades by its unprecedented compactness.

\section{Conclusions}

Plasmonics features unprecedented compactness in photonic integrated circuits due to strong confinement of electrical and optical fields to subwavelength dimensions in modulator and detector devices. Hereby, the devices exploit the unique properties of plasmonics like high bandwidth, dense parallelisation, energy-efficiency and flexibility for co-integration with electronics. The demonstrations of a $222 \mathrm{GBd}$ hybrid transmitter and a $120 \mathrm{GBd}$ monolithic transmitter are considered milestones towards an industrial use of plasmonics for optical communication. Hence, plasmonics is ready to tackle the current and future challenges in optical communication systems and might play a key role in overcoming the growing optical interconnect bottleneck.

\section{Acknowledgments}

This work was funded in part by the EC H2020 projects PLASMOfab (688166) and plaCMOS (980997).

\section{ORCID iDs}

Ueli Koch (DD https://orcid.org/0000-0001-8796-2146

1) P. J. Winzer and D. T. Neilson, J. Lightwave Technol. 35, 1099 (2017).

2) T. Alexoudi et al., J. Lightwave Technol. 37, 363 (2019).

3) C. A. Thraskias, E. N. Lallas, N. Neumann, L. Schares, B. J. Offrein, R. Henker, D. Plettemeier, F. Ellinger, J. Leuthold, and I. Tomkos, IEEE Commun. Surv. Tutorials 20, 2758 (2018).

4) R. Nagarajan et al., IEEE J. Sel. Top. Quantum Electron. 16, 1113 (2010) 
5) G. Santini, C. Caillaud, J. F. Paret, F. Pommereau, K. Mekhazni, C. Calo, and M. Achouche, Opt. Express 25, 25719 (2017).

6) S. Arafin and L. A. Coldren, IEEE J. Sel. Top. Quantum Electron. 24, 1 (2018).

7) J. M. Estaran et al., J. Lightwave Technol. 37, 178 (2019).

8) C. Wang, M. Zhang, X. Chen, M. Bertrand, A. Shams-Ansari, S. Chandrasekhar, P. Winzer, and M. Loncar, Nature 562, 101 (2018).

9) M. He et al., Nat. Photonics 13, 359 (2019).

10) J. Leuthold et al., IEEE J. Sel. Top. Quantum Electron. 19, 3401413 (2013).

11) X. Chen, M. M. Milosevic, S. Stankovic, S. Reynolds, T. D. Bucio, K. Li, D. J. Thomson, F. Gardes, and G. T. Reed, Proc. IEEE 106, 2101 (2018)

12) J. Witzens, Proc. IEEE 106, 2158 (2018).

13) K. Li et al., Optica 7, 1514 (2020).

14) C. Haffner et al., Nat. Photonics 9, 525 (2015).

15) M. Ayata et al., Science 358, 630 (2017)

16) Y. Salamin, P. Ma, B. Baeuerle, A. Emboras, Y. Fedoryshyn, W. Heni, B. Cheng, A. Josten, and J. Leuthold, ACS Photonics 5, 3291 (2018).

17) A. Messner, F. Eltes, P. Ma, S. Abel, B. Baeuerle, A. Josten, W. Heni, D. Caimi, J. Fompeyrine, and J. Leuthold, J. Lightwave Technol. 37, 281 (2019).

18) P. Ma, Y. Salamin, B. Baeuerle, A. Josten, W. Heni, A. Emboras, and J. Leuthold, ACS Photonics 6, 154 (2019).

19) U. Koch et al., J. Lightwave Technol. 37, 1484 (2019).

20) W. Heni et al., Nat. Commun. 10, 1694 (2019).

21) M. Burla et al., APL Photonics 4, 056106 (2019).

22) B. Baeuerle et al., Opt. Express 27, 16823 (2019).

23) W. Heni et al., J. Lightwave Technol. 38, 2734 (2020).

24) U. Koch et al., Nat. Electron. 3, 338 (2020).

25) Yole. SiPh Market Study (2020).

26) M. Nakamura, F. Hamaoka, M. Nagatani, Y. Ogiso, H. Wakita, H. Yamazaki, T. Kobayashi, M. Ida, H. Nosaka, and Y. Miyamoto, Presented at 2019 Optical Fiber Communications Conf. and Exhibition (OFC), 2019.

27) K. T. Settaluri et al., Presented at 41 st European Solid-State Circuits Conf. (ESSCIRC), 2015

28) R. Going et al., Presented at 43rd European Conf. on Optical Communication (ECOC), 2017.

29) A. H. Atabaki et al., Nature 556, 349 (2018).

30) V. Stojanovic et al., Opt. Express 26, 13106 (2018).

31) C. Uhl, H. Hettrich, and M. Möller, Electron. Lett. 56, 69 (2020).

32) U. Koch et al., Presented at 2020 Int. Conf. on Solid State Devices and Materials, 2020.

33) U. Koch, C. Hoessbacher, A. Emboras, and J. Leuthold, J. Electroceram. 39, 239 (2017).

34) E. Ozbay, Science 311, 189 (2006)

35) D. K. Gramotnev and S. I. Bozhevolnyi, Nat. Photonics 4, 83 (2010).

36) J. A. Schuller, E. S. Barnard, W. S. Cai, Y. C. Jun, J. S. White, and M. L. Brongersma, Nat. Mater. 9, 193 (2010).

37) T. W. Ebbesen, H. J. Lezec, H. F. Ghaemi, T. Thio, and P. A. Wolff, Nature 391, 667 (1998).

38) S. G. Rodrigo, F. D. Leon-Perez, and L. Martin-Moreno:, Proc. IEEE 104, 2288 (2016).

39) M. Moskovits, J. Chem. Phys. 69, 4159 (1978).

40) R. Pilot, R. Signorini, C. Durante, L. Orian, M. Bhamidipati, and L. Fabris, Biosensors 9, 57 (2019).

41) P. Berini, Phys. Rev. B 61, 10484 (2000)

42) G. Veronis and S. H. Fan, Opt. Lett. 30, 3359 (2005).
43) D. F. P. Pile, T. Ogawa, D. K. Gramotnev, Y. Matsuzaki, K. C. Vernon, K. Yamaguchi, T. Okamoto, M. Haraguchi, and M. Fukui, Appl. Phys. Lett. 87, 261114 (2005).

44) T. W. Ebbesen, C. Genet, and S. I. Bozhevolnyi, Phys. Today 61, 44 (2008).

45) R. F. Oulton, V. J. Sorger, D. A. Genov, D. F. P. Pile, and X. Zhang, Nat. Photonics 2, 496 (2008).

46) N. Kinsey, M. Ferrera, V. M. Shalaev, and A. Boltasseva, J. Opt. Soc. Am. B 32, 121 (2015).

47) Y. Fang and M. Sun, Light: Sci. Appl. 4, e294 (2015)

48) L. Novotny and N. van Hulst, Nat. Photonics 5, 83 (2011).

49) S. K. Patel and C. Argyropoulos, Epj Appl. Metamateria 2, 4 (2015).

50) S. Kasani, K. Curtin, and N. Q. Wu, Nanophotonics 8, 2065 (2019).

51) M. Bauch, K. Toma, M. Toma, Q. W. Zhang, and J. Dostalek, Plasmonics 9 , 781 (2014).

52) B. Spackova, P. Wrobel, M. Bockova, and J. Homola, Proc. IEEE 104, 2380 (2016).

53) J. R. Mejia-Salazar and O. N. Oliveira, Chem. Rev. 118, 10617 (2018).

54) T. T. Nguyen, F. Mammeri, and S. Ammar, Nanomaterials 8, 149 (2018).

55) M. Sharifi, F. Attar, A. A. Saboury, K. Akhtari, N. Hooshmand, A. Hasan, M. A. El-Sayed, and M. Falahati, J. Control. Release 311, 170 (2019).

56) L. Wang, M. H. Kafshgari, and M. Meunier, Adv. Funct. Mater. 30, 2005400 (2020).

57) A. M. Shrivastav, U. Cvelbar, and I. Abdulhalim, Commun. Biol. 4, 70 (2021).

58) A. Emboras, C. Hoessbacher, C. Haffner, W. Heni, U. Koch, P. Ma, Y. Fedoryshyn, J. Niegemann, C. Hafner, and J. Leuthold, IEEE J. Sel. Top. Quantum Electron. 21, 276 (2015).

59) T. J. Davis, D. E. Gomez, and A. Roberts, Nanophotonics 6, 543 (2017).

60) R. F. Oulton, V. J. Sorger, T. Zentgraf, R. M. Ma, C. Gladden, L. Dai, G. Bartal, and X. Zhang, Nature 461, 629 (2009).

61) Y. Liang, C. Li, Y. Z. Huang, and Q. Zhang, ACS Nano 14, 14375 (2020).

62) G. V. Naik, V. M. Shalaev, and A. Boltasseva, Adv. Mater. 25, 3264 (2013).

63) K. M. McPeak, S. V. Jayanti, S. J. P. Kress, S. Meyer, S. Iotti, A. Rossinelli, and D. J. Norris, ACS Photonics 2, 326 (2015).

64) J. C. Weeber et al., Opt. Express 25, 394 (2017).

65) D. F. P. Pile and D. K. Gramotnev, Appl. Phys. Lett. 89, 041111 (2006).

66) Q. A. Li, Y. Song, G. Zhou, Y. K. Su, and M. Qiu, Opt. Lett. 35, 3153 (2010).

67) D. Chelladurai, M. Doderer, U. Koch, Y. Fedoryshyn, C. Haffner, and J. Leuthold, Opt. Express 27, 11862 (2019).

68) M. Ayata, Y. Fedoryshyn, U. Koch, and J. Leuthold, Opt. Express 27, 29719 (2019).

69) A. Messner et al., Presented at 2020 Optical Fiber Communications Conf. and Exhibition (OFC), 2020.

70) W. Heni et al., Opt. Express 25, 2627 (2017).

71) C. Haffner et al., Opt. Mater. Express 7, 2168 (2017).

72) A. Melikyan et al., Nat. Photonics 8, 229 (2014).

73) C. Haffner et al., Nature 556, 483 (2018).

74) M. Ayata et al., J. Lightwave Technol. 37, 1492 (2019).

75) H. A. Atwater and A. Polman, Nat. Mater. 9, 205 (2010).

76) A. Dorodnyy, Y. Salamin, P. Ma, J. V. Plestina, N. Lassaline, D. Mikulik, P. Romero-Gomez, A. F. I. Morral, and J. Leuthold, IEEE J. Sel. Top. Quantum Electron. 24, 4600313 (2018).

77) A. Konczykowska, F. Jorge, M. Riet, V. Nodjiadjim, B. Duval, H. Mardoyan, J. M. Estaran, A. Adamiecki, G. Raybon, and J. Y. Dupuy, Electron. Lett. 55, 242 (2019).

78) M. Möller, T. F. Meister, R. Schmid, J. Rupeter, M. Rest, A. Schopflin, and H. M. Rein, Electron. Lett. 34, 1782 (1998). 